\title{
Prevención y discapacidad: modelo de gestión participativo
}

\section{Prevention and disability: participatory management system}

\section{Tomás Castillo Arenal', Rubén García Tejedor', Susana Delgado Mijares'}

1. AMICA. SAEMA. España.

\section{Correspondencia}

Rubén García Tejedor

AMICA. SAEMA. España.

Correo electrónico: rubeng@amica.es

\section{Resumen}

A partir del estudio de los accidentes del Grupo AMICA en los años 2006-2009 se determinó que ésta no era homogénea existiendo 2 puntos críticos:

- AMICA: la movilización de personas con discapacidad en los centros de alojamiento, suponía más del $50 \%$ de los accidentes ocurridos.

- SAEMA EMPLEO SL (Centro Especial de Empleo): aumento de la siniestralidad en un 50\% entre los años 2006 a 2009. Frente a la reducción producida en el Grupo AMICA.

Gerencia propuso desarrollar un plan de actuación específico para cada punto crítico, basado en la colaboración entre trabajadores y empresa, contando con el asesoramiento del ICSST.

La aplicación práctica se llevó a cabo creando un grupo de trabajo en cada entidad compuesto por:

- 2 delegados de prevención.

- 2 técnicos del servicio de prevención.

- 2 mandos intermedios.

- Dirección técnica de AMICA / SAEMA EMPLEO SL.

La dinámica de trabajo se estructuró realizando reuniones periódicas, en las que se analizaban los resultados de las medidas de mejora implantadas y se presentaban nuevas propuestas de actuación.

Grupo de trabajo AMICA: a partir de los resultados de la evaluación ergonómica realizada, se decidió actuar sobre la formación de los trabajadores y mejorar las ayudas técnicas disponibles en los centros de trabajo.

Grupo de trabajo SAEMA EMPLEO SL: se propuso mejorar la formación e información que reciben los trabajadores y revisar las condiciones de trabajo, actuando sobre las causas más frecuentes y las susceptibles de provocar los accidentes más graves.

Palabras clave: Discapacidad - Participación - Mejora continua - Liderazgo.

\section{Abstract IIIก}

From the study of accidents of AMICA's Group in the years 2006 - 2009, it was determined that the accident rate was not homogeneous, there were two critical points: accidents.

AMICA: moving and handling of disabled people in accommodation centers, was more than $50 \%$ of 
SAEMA EMPLEO S.L.: the accident rate increased by $50 \%$ between the years 2006-2009, against the reduction that took place in AMICA's Group.

Management proposed to develop a specific action plan for each critical point, based on the collaboration between workers and the company, with the advice of the ICASST.

The practical application took place by creating a working party on each entity. They were composed of:

2 safety workers.

2 safety technicians of the Preventive Service.

2 senior managers.

Technical manager of AMICA / SAEMA EMPLEO SL.

Working dynamics was structured by holding regular meetings, where the results of the implemented improvement measures were analysed and new proposals for action were presented.

AMICA working party: from the results of the ergonomic assessment, it was decided to act on the training of workers and to improve the technical aids available at the workplace.

SAEMA EMPLEO SL working party: the proposal was to improve the training and information that workers receive and to review working conditions, acting on the most frequent accident causes and on the most likely to cause the most serious accidents.

Key words: Disability - Involvement- Upgrade-Leader Ship. 


\section{INTRODUCCIÓN}

El Grupo AMICA tiene como principio y fin último la promoción y mejora continua de la calidad de vida de las personas con discapacidad. La seguridad y la salud en el puesto de trabajo debe ser un referente común para la diversidad de actividades desarrolladas por sus trabajadores: de apoyo a personas con discapacidad en centros de día, de formación, de alojamiento,... y en actividades productivas de lavandería industrial, confección, gestión de residuos, recuperación paisajística, mantenimiento de instalaciones, transporte adaptado...

Para ello, asumimos como propias las obligaciones indicadas en la ley 31/1995 de 8 de noviembre, de Prevención de Riesgos Laborales y su normativa de desarrollo. Este compromiso con la prevención y las responsabilidades que se deriven atañen a todos los niveles de actividad, siendo inviable sin la colaboración de todos y cada uno de los trabajadores.

Para la organización de los recursos preventivos se ha optado por un sistema mixto, que consiste en la creación de un Servicio de Prevención Mancomunado para el Grupo AMICA (SPM Grupo AMICA) y la contratación de recursos preventivos ajenos. Este modelo de trabajo y los medios con los que cuenta el Servicio de prevención, ha sido aprobado en el seno del Comité de Seguridad y Salud del Grupo AMICA el 30 de abril de 2010 y por la Dirección Gerencia el 26 de mayo de 2010.

El SPM Grupo AMICA asume como especialidades preventivas:

- La seguridad en el trabajo.

- La higiene industrial.

- La ergonomía y la psicosociología aplicada.

El Grupo AMICA tiene contratada la especialidad de Medicina del trabajo con un servicio de prevención ajeno. Así mismo podrá contratar actividades específicas que no se puedan asumir como elementos de gestión habitual.

El SPM Grupo AMICA actualiza el plan de prevención haciéndolo coincidir con el plan estratégico de la organización, estableciendo las líneas generales de actuación en materia preventiva.

El plan de prevención vigente tiene como objetivo reducir la siniestralidad el $12 \%$ en el periodo 2013-2016.

Desde la elaboración del primer plan de prevención en año 2004 en el que se partía de un índice de frecuencia de 28.93 accidentes por millón de horas trabajadas se ha pasado en 2012 a un índice de 14.77 lo que supone una reducción de aproximadamente del $50 \%$.

Los planes de actuación específicos para AMICA y SAEMA EMPLEO S.L se realizaron siguiendo la metodología propuesta por el proyecto "Objetivo Cero Accidentes de Trabajo en Cantabria” liderado por el Instituto Cántabro de Seguridad y Salud en el Trabajo.

Este proyecto se alinea totalmente con la política de calidad y prevención del Grupo AMICA al estar basado en los procesos de mejora continua. Se desarrolla en 5 fases que constituyen un ciclo completo:

- Autodiagnóstico Inicial

- Política y organización preventiva

- Evaluación de riesgos y planificación de la actividad preventiva.

- Implantación del sistema de prevención.

- Evaluación de la eficacia. 


\section{Aplicación práctica del proyecto}

Grupo de trabajo de AMICA: Determinó con exactitud el factor crítico de riesgo consistente en la movilización de personas con discapacidad, con grandes necesidades de apoyo, en los centros de alojamiento, que supone el 50\% de los accidentes.

Se realizó una evaluación ergonómica de los centros de trabajo, aplicando el método MAPO (Movilización Asistencial de pacientes Hospitalizados) en los centros de alojamiento especializado en la atención de personas con graves discapacidades físicas.

De los resultados de la evaluación se decidió actuar sobre la formación de los trabajadores y mejorar los medios técnicos disponibles en los centros realizando las siguientes actuaciones:

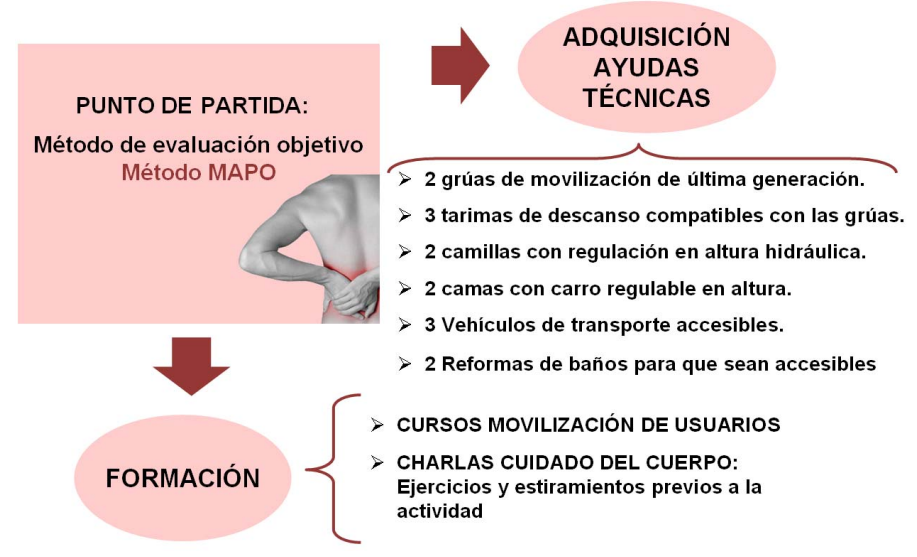

Grupo de trabajo de SAEMA: Estableció como causas principales del aumento de la siniestralidad, la puesta en marcha de nuevas actividades laborales en la recuperación y reciclado de envases, y el aumento de la plantilla en más de un $65 \%$. Esto produjo una reducción de la cultura preventiva, de la experiencia en el puesto de trabajo y del conocimiento de los riesgos de las actividades.

Desde el grupo de trabajo se propuso mejorar la formación e información que reciben los trabajadores. Se procedió a actualizar todas las evaluaciones de riesgos y se elaboraron manuales de información accesibles de cada puesto, que se entregaron a todos los trabajadores. Estos manuales se entregan en la incorporación al puesto de trabajo.

Se incidió en mejorar las condiciones de trabajo actuando sobre las causas más frecuentes y las susceptibles de provocar los accidentes más graves.

Las actuaciones sobre las causas más frecuentes de accidente (caídas al mismo nivel y los sobreesfuerzos) fueron:

- Recogida de lixiviados de la prensa dirigiéndolos hacia alcantarillado.

- Recogida de las pérdidas de aceite de lubricación de las cintas transportadoras.

- Creación de caminos de seguridad en la planta.

- Unión de las rejillas del alcantarillado evitando que se levanten eliminando el riesgo de tropiezos.

- Instalación de pasarela de acceso seguro a través del foso de alimentación.

- Adquisición de container para almacenar la chatarra reduciendo el número de manipulaciones.

- Adquisición de contenedores autovolteadores con ruedas para manipular los finos y chatarra por medio de carretilla elevadora.

— Limitación de la manipulación de cargas a $15 \mathrm{~kg}$. 
- Colocación de una tolva para bajar la chatarra desde los triajes.

- Sustitución progresiva de jaulas antiguas por equipos nuevos que se pueden mover por medio de la carretilla elevadora.

- Adquisición de apilador manual para palets.

Las actuaciones sobre los posibles accidentes más graves (caídas al interior de las prensas, caídas a distinto nivel y atropellos) fueron:

- Desarrollar a través de antenas de radio frecuencia y emisores personales un sistema de parada de emergencia de las prensas.

- Colocar barandillas y plataformas de trabajo en todo el perímetro de las cintas transportadoras.

- Limitar la velocidad a $10 \mathrm{~km} / \mathrm{h}$ en toda la planta.

- Adquirir una nueva carretilla elevadora y limitar electrónicamente su velocidad. Equipo dotado de puertas y cinturón de seguridad con bloqueo del equipo para evitar el atrapamiento del conductor por vuelco de la carretilla.

- Limitar el número de camiones que pueden descargar simultáneamente.

- Mejorar la uniformidad a través de elementos de alta visibilidad.

- Limitar el acceso a planta, de vehículos, con una barrera automática.

\section{Resultado del proyecto}

La aplicación de la metodología propuesta en el Proyecto Objetivo Cero Accidentes de Trabajo en la organización nos ha permitido:

- La participación de los delegados de prevención en los grupos de trabajo.

- Una mayor implicación de los mandos intermedios, por medio de la realización de propuestas de medida de mejora así como su implantación y verificación.

- El reconocimiento interno y externo a través de la presencia en medios de comunicación y actos públicos.

- Una mejora del clima laboral de los trabajadores al fomentar su participación activa en la mejora de las condiciones trabajo.

- La actualización del Sistema de Gestión de la Prevención con el apoyo y asesoramiento de los técnicos del ICASST.

- La reducción de la siniestralidad de la organización pasando en 2009 de un índice de frecuencia de 19.82 a 14.77 en 2012, lo que supone una reducción del $26 \%$.

Las medidas implantadas por los grupos de trabajo nos han permitido controlar los puntos críticos, cuantificando su eficacia con los siguientes indicadores:

- AMICA: En el trienio 2010-2012 se ha producido 1 accidente con baja como consecuencia de la movilización de usuarios, frente a los 6 ocurridos en los cuatro años anteriores. Lo que supone una reducción del 78\%.

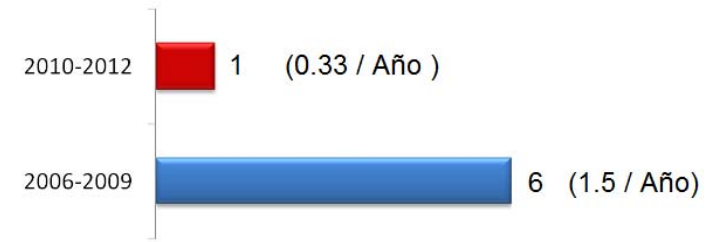


- SAEMA EMPLEO SL: Reducción del Índice de Frecuencia. En 2012 el índice ha sido de 10.01 con una reducción del 80\% del registrado en 2009.

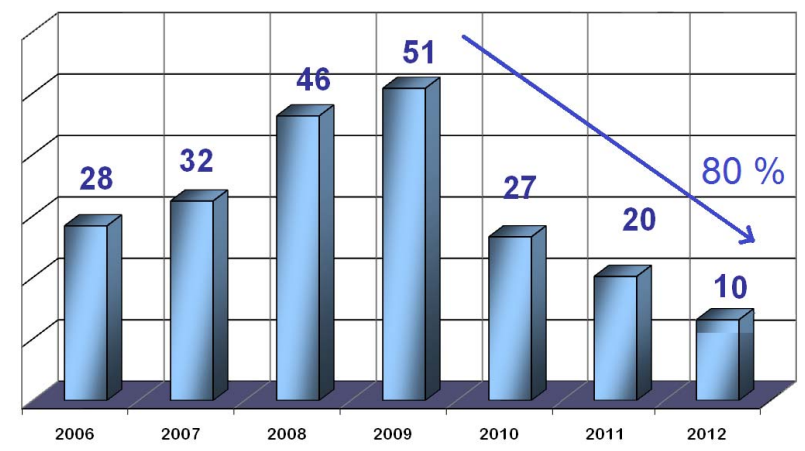

\section{Factores de éxito}

1. El liderazgo ejercido por los diferentes órganos de dirección del Grupo AMICA a lo largo de todo el desarrollo del proyecto.

2. La participación continúa de los trabajadores con discapacidad través de los grupos de trabajo.

3. La creación de grupos de trabajo específicos para abordar cada punto crítico, al constituir cada uno de ellos problemáticas totalmente diferentes.

4. El desarrollo e implantación de más de 25 actuaciones concretas destinadas mejorar las condiciones de trabajo (Un problema - una solución).

5. Demostrar que las personas con discapacidad no presentan una mayor siniestralidad que el resto de los trabajadores si el modelo de Gestión de la Prevención, basado en el liderazgo de la dirección y la mejora continua, les permite participar activamente.

\section{AGRADECIMIENTOS}

Miembros del Comité de Seguridad y Salud (CSS) de AMICA.

Miembros del CSS de SAEMA EMPLEO S1.

Instituto Cántabro de Seguridad y Salud en el Trabajo (ICASST), Amalio Sánchez Grande, Pedro Obregón Cagigas y Ana Breñosa Álvarez de Miranda. 